\title{
Particle tracking in a Two-Section Two-Zone Fluidized Bed Reactor (TS-TZFBR). Experimental technique and CCBM model validation
}

\author{
I. Julián, J. Herguido, M. Menéndez \\ Catalysis, Molecular Separations and Reactor Engineering Group (CREG), \\ Instituto de Investigación en Ingeniería de Aragón (I3A) \\ Universidad de Zaragoza, Mariano Esquillor s/n, 50018, Zaragoza, Spain. \\ Tel.+34 876555168, e-mail: ijulian@unizar.es
}

\begin{abstract}
The Two Zone Fluidized Bed Reactor (TZFBR) provides a high level of process integration, allowing reaction and, in situ, continuous catalyst regeneration, in a single fluidized bed. Reactive and regenerative atmospheres are induced simultaneously inside the single vessel due to a separated gas inlet and particles fluidization provides reactor continuous operation. Therefore, the fluid dynamic key factor in a TZFBR is the mixing rate between solids inside the two bed zones (a good mixture provides continuous catalyst regeneration, while bad contact between bed zones leads to deactivation and loss of catalytic surface). In the present study, phosphorescent particles have been used as optical tracers to measure solids axial mixing between reactor zones in a catalytic TZFBR. Additionally, a different cross-sectional area between zones has been studied to get a certain fluidization regime, allowing small flowrates in the regeneration zone. This geometry implied a transition angle $\alpha$ between zones to be implemented (Two-Section TZFBR). In line with it, a modified counter-current backmixing model (CCBM) without fitting parameters was developed to predict mixture rates inside the bed for different TS-TZFBR geometries. Modifications carried out in the CCBM basis model involved reactor geometry and the presence of two simultaneous gas feed points along the bed. Model parameters related to bubble/solid fraction and wake-emulsion mass transfer were fully correlated to operational conditions (gas velocity, feed point location and section change). The adapted CCBM model predictions were further validated with optical tracers experimental fluid dynamic data, resulting in a high agreement.
\end{abstract}

